Tery satisfactorily; the temperature went down at once, and she was not troubled with sickness. She passed $16 \mathrm{oz}$. of urine the night after the operation, of sp. gr. 1023, acid, and containing no albumin, but 1.2 per cent. of urea. On the second day she passed $16 \mathrm{oz}$. of dark chocolate-coloured urine (that passed both before and after being quite normal), which on skilled examination was said to be "deeply coloured by hæmoglobin, with dense deposit of blood, mucus, pus, \&c., innumerable crystals of calcium phosphate, some casts. numerous red blood-corpuscles, leucocytes in every stage of transition, and some renal epithelium." A smaller quantity of this type was again passed on the third day, but on no other occasion. The stitches were removed on the eighth day, when the wound was practically healed. She was keeping so well and improving so markedly in general health that, although the second cyst was notably increasing in size, whilst carefully watching her, I was disposed to wait Fet a few days ere submitting her to the second operation, which probably meant a nephrectomy. On the night of the sixteenth day from her first operation the temperature began to rise, but she herself seemed well, and the cyst, which had been still slowly increasing in extent, gave rise to no pain or tenderness, the urine, too, keeping normal. On the night of the eighteenth day it somewhat suddenly reached $104^{\circ}$. On the day following (June 25th), with the aid of the same assistants as before, I adopted the lumbar incision recommended by Mr. Henry Morris, and easily came down upon the left kidney and a somewhat tough-walled sac, closely approximated; the cyst was slightly connected with its surroundings by recent adhesions. Breaking these carefully down with the finger, I at length found that the cyst and kidney were entirely separable, being in no way connected. Having tapped the cyst with an ascites trocar and removed four and a half pints of dark greenish fluid, I without the least difficulty shelled it out from its bed. There was very little hæmorrhage, only one ligature being called for, and that in the abdominal wound. A large-sized rubber drainage-tube was put in deeply and the wound closed with silkworm gut. Save for the small aperture made by the trocar, the cyst proved watertight; its inner surface, over about a third of its extent, was coated with a thick, shaggy lining of recent organised lymph. The patient's recovery from the day of operation was uneventful. The temperature became normal on the fifth day and never rose again; she passed three pints of urine of normal character within the first thirty hours, and it has continued so since. The wound discharged very freely for two days and then gradually slackened. The stitches were removed on the fourth day, as was also the tube, as the edges of the wound showed some slight redness, which, however, then at once disappeared. At the end of eight days she was removed to a sofa in the daytime, and she is now convalescent and has gained flesh markedly.

Remarks.-The chief interest in this case centres round the retro-peritoneal cyst. I have carefully searched the literature at $m y$ disposal and cannot note a like case. It appeared on palpation (and the rare opportunity of effecting this intra- as well as extra-abdominally here existed) to be of renal origin; the character the urine presented, too, on the second and third day after the ovariotomy pointed seemingly to grave kidney dista bance. The position of the tumour and its consequent capability of acting as a kidney irritant or mere mechanical obstruction to the ureter must be borne in mind. It is also a singular fact that these two tumours should have been present at the same time in the same patient without giving rise to earlier or more marked symptoms.

Ptillimore-gardens, W.

The Hants County Asylum.-A new block for idiots has just been completed at the Hants County Asylum at Knowle, near Fareham. It is already occupied by thirtyseven patients. During the past year a committee of the county council have had under consideration the question of installing a water-softening apparatus to deal with the hard water which is obtained from the new pumping station, and a scheme for the carrying out of the work is promised for the next meeting of the county council. The separation financially of the Isle of Wight from the county of Hampshire proper has just been carried out, and in future patients from across the Solent will be charged at the same rate as other out coanty inmates of the asylum. There has been an increase, in common with the country as a whole, in the numbers of inmates, the total at present being 1083-492 males and 591 females.

\section{SEQUEL OF A CASE TREATED BY KOCH'S TUBERCULIN, WITH THE RESULTS OF THE NECROPSY.}

By J. G. SINCLAIR COGHILL, M.D., F.R.C.P. EDIN., SENIOR PHYSICIAN, ROYAL MATIONAL HOSPITAL FOR CONSUMPTION, VENTYOK.

IN $1891^{1}$ I described in detail the successful treatment of ten cases of pronounced pulmonary tuberculosis by Koch's method in the Royal National Hospital for Consumption, Ventnor, during a period of sixteen weeks. These cases were repeatedly examined by, and the treatment was conducted under the observation of a large number of, medical men unconnected with the hospital. 'The subsequent history of these cases has been carefully noted, and reports of their condition obtained at frequent intervals from competent observers. At present, after a lapse of over four years, eight of these ten patients are in excellent health, with every evidence of complete arrest of the pulmonary lesion, and are following their ordinary avocations. One of them has been serving for nearly three years in one of the battalions of Her Majesty's Foot Guards. Of the other two cases, onereferred to in my report as No. 7-died eighteen months after leaving the hospital from a return of symptoms referable to fæcal accumulation, similar to that which cansed the tuberculin inoculations at the hospital to be suspended from the 19th to the 31st March. This attack was in nowise associated with tuberculosis.

The remaining case, described in my report as No. 10, was inoculated, I may say, almost under protest, both lungs being affected, the tuberculous process so acute, advanced, and extensive, and the general conditions also most unfavourable. He pleaded so earnestly that as he had only a few months, perhaps weeks, to live, he might be allowed the chance of improvement for the sake of his wife and young children, and I must confess I very reluctantly yielded. After leaving the hospital the patient took up his residence in Ventnor. The case was therefore kept continuously under my own observation and examined from time to time until the fatal termination, which occurred very suddenly from cardiac failure on Nov. 17th, 1894. A post-mortem examination was made within eighteen hours, and it is most interesting to compare the conditions then ascertained, with the physical signs and other symptoms described in my report as previously noted during and after treatment by tuberculin innoculation. For the purpose of this comparison I must briefly recapitulate the principal details of the case from the above mentioned report. 'The patient was a man aged forty-five years with a strumous diathesis. His height was $5 \mathrm{ft} .5 \frac{1}{4}$ in., and weight 8 st. 4 lbs. He had been ill for three years. Severe and persistent cough began two years previously. There had been night sweats for twelve months, with loss of appetite, and progressive emaciation. There had been no hæmoptysis. Before innoculation he expectorated daily an average of two ounces of thick, greenish-yellow sputum, nummular, and containing aburdant bacilli. The evening temperature reached $101^{\circ} \mathrm{F}$., and in the morning was subnormal. The specific gravity of the urine was 1022, acid, with a slight mucous deposit, and a haze of albumin. The pulse was 102, soft and compressible. The first cardiac sound in the mitral area, and the second sound in the pulmonary area, were reduplicated. Respiration was 25 per minute. The physical signs in the lungs were as follows. There was flattening at both apices, above and below the clavicle, but more so on the left side, where movement was also more restricted. On the right side in front the percussion note was impaired to the third rib, with expiration prolonged, vocal resonance increased, and distant amphoric breathing under the clavicle. Behind, the note was impaired at the apex, expiration was lengthened, and increased vocal resonance to spine of scapula. In front of the left lung there was absolute dulness to the third rib and impaired to the fifth rib. There was loud superficial cavernous breathing with pectoriloquy to the lower margin of the third rib, with moist crepitations over the whole lung. Posteriorly there was absolute dulness at the apex and in the supraspinous fossa; in the latter there was amphoric breathing but no crepitation. 
The patient was inoculated with 0.001 gramme of tubcrculin at 10 A.M. on Dec. 27th, 1890 ; the highest temperature was $102.5^{\circ}$ at 9 P.M. The pulse was 116 . The breath rate was unaffected, but the cough was severe, and the expectoration, which was very frothy, increased to thrce ounces in twentyfour hours. The urine became flocculent on cooling and showed a phosphatic deposit with alkaline reaction and sp. gr. 1020 . On examining the chest, in addition to the previously noted sign, fine crepitations could be heard all over the left lung posteriorly. By the 29th these crepitations had disappeared, and the patient stated that he he "had coughed up less the last twenty-four hours than for the last two or three months," but bacilli were still found even more thickly clustered in the sputum. By Jan. 5th, 1891 (nine days), he had lost $41 \mathrm{~b} .4 \mathrm{oz}$, and this loss of weight occurred during the course of the earlier inoculations in seven of the ten patients under treatment. From this I inferred that the dose of tuberculin was still too large, and the reactions injuriously excessive, and in all subsequent inoculations I adapted the amount of tuberculin, so as to produce merely an indication of reaction, and to this I mainly attribute the exceptional success of the treatment in my hands. By Jan. 19th the loss of weight was recovered, and the increase went on rapidly and continuously. On Feb. 8th the fifth inoculation of 0.003 gramme was followed by moist râles or crepitations, exaggerated on coughing, over the whole of the right lung in front and the upper'third behind; and in the left lung anteriorly there were crepitations over the base and friction round the lower limits of the vomica; at the apex behind there were also crepitations. The development from time to time after inoculation of peculiarly superficial, small, dry crepitations, especially over the bases of the lungs posteriorly, was noted in nearly all the cases. These crepitations conveved to the ear the impression of some pleuritic origin, as if the pleura was hyperæmic or the pulmonary tissue immediately under it was affected in some way. The association, also frequently noted after the earlier and larger inoculations, especially of severe pains in the joints, abdomen, and head would point to some similar concurrent cause, but not, however, of a tuberculous nature. Their disappearance was usually as sudden as their onset and as complete. By April 14th this patient had had twenty-two inoculations-the highest, 0.065 gramme-amounting in all to 0.4625 gramme of tuberculin. The greatest reaction, $104.6^{\circ}$, was reached on Jan. 8th, after 0.002 gramme In this case I was much impressed with what seems almost to amount to a law-that, in proportion to the extent and activity of the tuberculous lesions, the greater and more prolonged is the reaction to the same doses of the lymph; hence, the more advanced and active the pulmonary lesion the greater the necessity for proportionally smaller doses and longer intervals in its administration. On leaving the hospital on April 16th, 1891, his weight was 9 st. $31 \mathrm{lb}$, being a gain of $13 \mathrm{lb}$. since Dec. $25 \mathrm{th}, 1890$, and a gain of $17 \mathrm{lb} .4 \mathrm{oz}$. since Jan. 5th. The patient had improved remarkably in condition and appearance. He expressed himself as "feeling wonderfully better." He had no night-sweats. The cough was easier; but the expectoration varied greatly, and on the whole was less in quantity and not so purulent and nummular. Bacilli, however, were still abundant. The appetite and digestion were excellent, and the functions regular. His pulse was stronger and fuller. The first sound of the heart was reduplicated in the mitral, and both sounds were reduplicated in the aortic and pulmonary areas. The flattening of the lungs was more marked, and morement more deficient under both clavicles, especially at the left side. In the right lung, anteriorly, the note was dull to the nipple. There was increased vocal fremitus, amphoric breathing under the clavicle, and pectoriloquy, with a few scattered crepitations; and posteriorly dulness to the inferior angle of the scapula with a very few crepitations. In the left lung the note from the apex to the nipple was more impaired than on the right side, with loud superficial amphoric breathing and pectoriloquy, with crepitations only on coughing, also a few small crepitations at extreme base. Posteriorly there was dulness to the spine of the scapula, obscure amphoric breathing and exaggerated vocal resonance in the supra-spinous fossa, and towards the base a few scattered crepitations. The patient, with his family, took up his residence in Ventnor. I inoculated him at intervals for six weeks, by which time the temperature, now normal, ceased to respond to the tuberculin. The moist crepitations throughout the lungs gradually cleared up, and the other physical signs indicated that only the smaller vomica at the right apex was quite dry, the larger at the left apex still affording moist crepitations on coughing. The only cough was that necessary to raise daily, and mostly in the morning, the six to eight nummules which now constituted his daily expectoration. The patient, who could be seen daily walking smartly about Ventnor and the neighbourhood, came to me every three months or so to report himself and to be ex. amined. The sputa always contained bacilli, the physicas signs remained the same except when an occasional catarrt declared itself, which, however, always passed off. The patient all the time used a respiratory inhaler with guaiacol, eucalyptol, and chloroform, and took, with occasional interruptions, capsules of five minims of guaiacol (three daily), which he said "always improved his wind." on Sunday Dec. 11th, 1894, he had been at morning service and hac afterwards partaken of a hearty dinner at table with his family. On the 13th I was asked to see him "as his breath. ing was very bad." I found him suffering from extreme dyspnoca, with an anxious, drawn face, but able to speak rapidly and explain his symptoms. The pulse was hardly to be counted, it was so faint and compres. sible. The heart sounds were also very faint, and the apex beat was hardly perceptible. I prescribed a mixtare of tincture of cligitalis, tincture of sumbul, ether, chloroform, and compound tincture of cinchona co., to be given at frequent intervals, with appropriate nourishment, \&c. Next day found him very much better. I visited him the next day but one, when he seemed further improved. The following day I also saw him, and he wrote out some directions and signec some documents with a steady hand, but I had not left him above an hour when he all at once raised his hands laid them on his wife's shoulders, and then swddenly expired.

Necropsy. - The post-mortem examination was performec eighteen hours after death by Dr. Lewis and Dr. Frazer, resident medical officers of the Royal National Hospital at Ventnor. They made independent notes which are embodied in this report. The body was found to be fairly well nourished. The right lung was adherent, and the pleura much thickened over the upper half of its extent; the left lung was adherent superiorly for about two-thirds of its extent, and there were also smaller adhesions to the pericardium and dia. phragm. There was, however, no difficulty in removing the lungs from the thoracic cavity without rupture of their substance. The whole of the upper lobe of the left lung was occupied by a cavity with thick fibrous capsule and lined by a pyogenic membrane with pus-moistened walls. The middle lobe was adherent to the upper and also entirely excavated. and the capsule and lining presented similar characters. These vomicæ did not communicate, and each opened indeperdently into a bronchus. The right lung contaimed a caseous nodule surrounded by a thick fibrous capsule. The: whole upper third of this lung was occupied by a cavity with an extremely thick fibrous wall. The lining membrane bac no pus on its wall-indeed, it seemed almost dry; it com municated with a small bronchus. This vomica is the one recognised in the original examination and referred to in the report as of older standing than the excavation in the left apex, and as also being in a condition of arrest. The bases of both lungs were much congested hypostatically, and contained a few capsulated caseous nodules scattered throughout their substance. The heart was extremely small, anæmic and its walls much thinned; the right side was dilated anc full of blood. The valves were quite heaithy.

Remarks.-If the physical signs and symptoms of this patient as ascertained by competent observers before treat ment, the phenomena dereloped during its course, and his condition at its termination, and subsequently, be compared with the state of the lungs as revealed at the necropsy, there can hardly be any hesitation, I think, in ascribing the arrest of this extensive area of acute pulmonary tuberculosis to judicious tuberculin inoculation. This case confirms me in the opinion I have always held, and still hold, that tuberculin has potent therapeutic efficacy in the treatment of tuberculosis when used judiciously and with caution in doses and under conditions adapted to each individual case. O the Continent Koch's method has survived the unscientific and regrettable haste which, after the great promise of its inception, too prematurely condemned it. No less a authority than Dr. Armand Ruffer, when writing to me lately on another subject, told me that he was mucb impressed with what he saw of this treatment on the occasion of a recent visit to Berlin.

Ventnor, Isle of Wight. 


\section{EXOPHTHALMIC GOITRE. ${ }^{1}$}

BY JOHN HILL ABRAM, M.D. LOND., M.R.C.P., ASSISTANT PHYSICIAN, STANLEY HOSPITAL; ASSISTANT TO THH PROFESSOR OF PATHOLOGY, UNIVERSITY COLLEGE; PATHOLOGIST, ROYAI INFIRMARY, LIVERPOOL.

DuRING the last two years I have had under my observation five cases of exophthalmic goitre, and I have had the exceptional experience of two necropsies-one on a case of my own and the other on a case of Dr. Davidson at the Infirmary. I shall divide the remarks I have to make into three groups-viz., (1) the symptoms; (2) the morbid anatomy and pathology ; and (3) prognosis and treatment.

1. Symptoms.-The onset may be acute or chronic, the course short or prolonged over years. The earlier symptoms are associated with the cardio-vascular system and consist chiefly of a persistent tachycardia. Occasional attacks of palpitation are present. Physical examination reveals a diffused cardiac impulse, with enlargement of the area of cardiac dulness. Functional bruits are common. The arteries in the neck are dilated, and visible pulsation in them is common. The radial pulse is frequent and of low tension; the frequency may be 150 to 200 per second. Goitre follows and consists of a firm enlargement of the gland, usually more marked upon the right side. The goitre is painless, and, in my experience, is not pulsatile. A rough systolic murmur is usually audible in the neighbourhood of the gland. Exophthalmos may be a very late symptom. other eye symptoms are defective descent and retraction of the upper lid. In one case I noted a symptom first recorded by Joffroy-viz., a loss of the contraction of the frontalis muscle associated with the sudden upward movement of the eyes. The pupils in my cases, with one exception, were not dilated. To the so-called cardinal symptoms another should be added-viz., muscular tremor. It was present in all my cases, and is, I believe, a constant and early symptom of the disease. The tremor has been thoroughly investigated by Marie, ${ }^{2}$ who states that it may be best brought out by getting the patient to hold the arms in the extended position. The narrow excursions and the rapidity ( $8 \frac{1}{2}$ per second) distinguish the tremor from other forms; that of alcoholism, which most resembles it, may be recognised by the irregularity both of time and excursions. The general features of the disease are familiar, and may be, therefore, very briefly given. They are emaciation, anæmia, irregular rises of temperature, flushings and sweatings, diarrhœa, and excitable mental state, passing on to actual insanity. Amenorrhcea is a constant feature in women. Diminution of the electrical resistance of the skin is said to be a constant phenomenon. In a few cases general enlargement of the lymphatic glands has been observed, and in both the necropsies I have made the lymphoid follicles in the intestines were swollen. Dr. Hunt informs me that he has twice operated for enlarged tonsils in cases of Graves' disease. In both cases the enlargement took place after the onset of the latter. Rarer symptoms are various forms of paralysis, glycosuria, albuminuria, and cedema. The termination may be in recovery or death from exhaustion or syncope.

With this brief account of the clinical features $I$ pass on to consider my second group.

2. Morbid anxtomy and pathology.-The older theories of Stokes and Graves (primary cardiac) and Marshall and Taylor (pressure on nerves and vessels of neck) have been long abandoned. The sympathetic theory must, I think, now be given up also. The tachycardia and the vascular dilatation at first sight suggest a lesion of the sympathetic nervous system. On closer examination it is found, as Gowers points out, ${ }^{3}$ that this theory involves the assumption of $(a)$ a partial affection, or $(b)$ a twofold action-viz., a stimulating one upon the accelerating fibres for the heart and a paralysing one upon the vaso-motor fibres-a double-barrelled action the probability of which is rather remote. Again, if there were a sympathetic lesion we should expect dilatation of the pupils to be a common symptom, whereas it is rare. It was only present in one of

${ }^{1}$ A paper read before the Medical Institution of Liverpool on 0ct. 17th, 1895

${ }^{2}$ Contribution à l'Étude des Formes frustes de la Maladie de Basedow. Paris, 1883.

Diseases of the Nervous System, vol. ii., p. 885 my five cases. Moreover, the sympathetic system in the neck has been found to be normal in the vast majority of the fatal cases. In thirteen cases recorded by Lewin ${ }^{4}$ it was normal in all. It was normal in both my cases. A much more probable theory is one which locates the lesion in the central nervous system, and more especially in the medulla. The association of arterial dilatation with increased frequency of the heart's action is in harmony with Marey's well-known law that "there is an inverse ratio between pulse frequency and blood pressure in consequence of their relationship to a central mechanism." Filehne, and Durduf, in repeating Filehne's experiments, have shown that division of the restiform body gives rise to symptoms closely resembling those of exophthalmic goitre. There is no doubt, too, that in some cases, more especially the acute ones, lesions have been found in the medulla-e.g., increased vascularity by Greenfield ${ }^{\bar{\sigma}}$ and myself, hæmorrhages by Müller, ${ }^{6}$ Hale White, Stewart and Gibson, ${ }^{7}$ and abscess by Stewart and Gibson. ${ }^{8}$ The usual absence of anatomical changes and the undoubted presence of a marked neurotic inheritance led Charcot to regard the disease as a neurosis, and to this phase of the nervous theory I shall allude again. For my own part, I fail to see how a lesion in the medulla can give rise to the psychical and paralytic symptoms so commonly met with in this disease. It is probable that the cardio-vascular phenomena are due to a disturbance of the medullary centres, but that this disturbance is secondary and not primary. The nervous system theory is still accepted by many writers (Mannheim ${ }^{9}$ and others), but the latest, and at present, I think, the most satisfactory, theory is the one which attributes the disease to a primary lesion of the thyroid gland. In considering the affection from this aspect the thyroid gland must be dealt with first. The examination of the goitre in my two cases revealed a condition which was in striking contrast to that usually described. The condition I found corresponds closely to that recorded as present in their cases by Stewart and Gibson, ${ }^{10}$ Greenfield, ${ }^{11}$ O. Hezel, ${ }^{12}$ Joffroy, ${ }^{13}$ and Putna $\mathbf{m} .{ }^{14}$ The thyroid gland was enlarged; the alveoli were small and apparently more numerous, they were packed with cells, and the colloid material was absent. In one of my cases (acute) there were masses of leucocytes at the points of entrance of the larger vessels. In chronic cases an overgrowth of connective tissue has been present, with some tendency to cyst formation. It will be noticed that in this description there is no allusion to any marked increase of vascularity. Is the goitre a vascular one? From the clinical side I must say that in my five cases repeated examinations failed to detect any expansile pulsation in the goitre, and the histological examination of my own case and under Dr. Davidson's care confirms me in the belief that the goitre is not a vascular one. Of course, some increase in vascularity is necessarily present in an over-active gland, but my point is that there is nothing approaching an aneurysmal condition, and therefore the goitre in Graves' disease is not clinically $a_{\text {: }}$ pulsatile one. ${ }^{1 \overline{5}}$ The marked thrill and bruit present in these cases are due to vibrations in the main thyroid arteries and not in the gland itself. It is important to note that the histological characters described above are present even when the gland during life is not appreciably enlarged-a point of great interest in connexion with cases of persistent tachycardia, which many observers would class as fruste examples of Graves' disease. Greenfield ${ }^{16}$ compares the histological characters to those seen in an active mammary gland, and suggests that the thyroid gland in these cases is in a state of over-activity. Some support is lent to this suggestion by the fact that the gland tissue in Graves' disease bears a close resemblance to the thyroid tissue in early life, when the gland is supposed to be in a state of great activity. ${ }^{17}$ Assuming, as I think is permissible, that the thyroid gland

4 Inaugural Diszertation, Berlin, 1888

5 Bradshaw Lectures, THE La ANET, Dec. 16th, 1893.

6 Deutsches Archiv für Klinische Medicin, Baud xli.

7 Brit. Med. Jour., Sept. 23rd, 1893. 8 Ibid. 9 Morbus Gravesii. Berlin, 1894.

10 Brit. Med. Jour., Sept. 23rd, 1893. II THE: LANCET, Dec. 16th, 1893.

12 Deutsche Zeitschrift für Nervenheilkunde, 1873.

13 Progrès Médical, December, 1894.

14 American Journal for Nervous and Mental Diseases, December, 1893 15 My point is sustained by a case of Mr. Paul, in which, when the isthmus was divided in the operation of partial thyroidectomy for Graves' disease, no bleeding of any mnment took place. 16 The LanckT, Dec. 16th, 1893

1) Professor Sherrington informs me that after partial thyroidectomy the remaining gland tissue assumes the character of that seen in Graves' disease-a fact which gives additional and strong support to Graves disease-a fact
Greenfield's suggestion. 